\title{
Kinematical data on early-type galaxies. IV. ${ }^{\star, \star \star}$
}

\author{
F. Simien and Ph. Prugniel \\ CRAL-Observatoire de Lyon, CNRS UMR 142, F-69561 St-Genis-Laval Cedex, France
}

Received February 4; accepted March 9, 1998

\begin{abstract}
We present kinematical data for a sample of 25 galaxies. Rotation curves and velocity-dispersion profiles are determined for 16 objects, while the central velocity dispersions are given for the whole sample. This is our fourth paper in a series devoted to the presentation of kinematical data on elliptical and S0 galaxies, derived from long-slit absorption spectroscopy.
\end{abstract}

Key words: galaxies: elliptical \& lenticular, $\mathrm{cD}$ galaxies: kinematics and dynamics - galaxies: fundamental parameters - galaxies: general

\section{Introduction}

We have recently begun to present kinematical measurements from absorption spectroscopy on early-type galaxies (Simien \& Prugniel 1997a,b, and c: hereafter Papers I, II, and III, respectively); these data are intended to contribute to the study of several structural and evolutionary issues. As part of our continuous effort to get reliable velocity dispersions and rotation curves on a statistically significant sample of objects, we presently report on observations on a fourth list of targets.

This work follows closely the technique already described in full detail in Paper I, for both observation and reduction, and only a minimum of explanations will be given here.

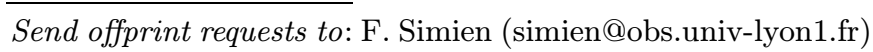

* Based on observations collected at the Observatoire de Haute-Provence.

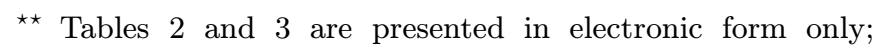
Tables 1 through 3 are available from the CDS, Strasbourg, via anonymous ftp to cdsarc.u-strasbg.fr (130.79.128.5) or via http://cdsweb.u-strasbg.fr/Abstract.html

\section{Sample and observations}

Our present sample consists of 24 early-type galaxies, with 20 ellipticals and four S0s, and one spiral. There is only one object in common with the preceding papers of the series: NGC 4318, which appeared in Paper II; this duplicate measurement has been included in the present work for comparison purposes. Relevant catalog elements are presented in the first 10 columns of Table 1. The Es have ellipticities corresponding to classes between $\simeq \mathrm{E} 1$ and $\simeq \mathrm{E} 5$, and the S0s are moderately to highly flattened. The distances are in the range of $\simeq 10$ to $\simeq 74 \mathrm{Mpc}$ (for $\left.H_{0}=75 \mathrm{~km} \mathrm{~s}^{-1} \mathrm{Mpc}^{-1}\right)$. These objects are intrinsically bright $\left(-22.0<M_{B}<-18.0\right)$, except for NGC 4239 and NGC 4318, which are significantly fainter.

The observations have been secured at the $1.93-\mathrm{m}$ telescope of the Observatoire de Haute-Provence, equipped with the CARELEC long-slit spectrograph. The selected setup provided a spectral range of either $\simeq 900 \AA$ (with a dispersion of $1.8 \AA$ per pixel at $\lambda=5200 \AA$, i.e. $104 \mathrm{~km} \mathrm{~s}^{-1}$ ), or $\simeq 450 \AA$ (with a dispersion of $0.9 \AA$ per pixel, i.e. $52 \mathrm{~km} \mathrm{~s}^{-1}$ ). The slit width, projected onto the plane of the sky, was $2.2^{\prime \prime}$.

In February, October, November 1992, and in March 1993, four observing runs totalling nine clear nights allowed us to collect one or two spectra on these 25 galaxies. For 20 of them, the slit was positioned on the major axis. For NGC 2872, paired with the spiral NGC 2874, the slit was oriented along the direction linking the two objects, and we included the central velocity dispersion of NGC 2874 in Table 1. For IC 4051, NGC 2476, and NGC 3605, the adequate PA values were unfortunately missing in our files, and we adopted either arbitrary values $\left(0\right.$ or $\left.90^{\circ}\right)$, or old, unaccurate determinations.

The atmospheric conditions were variable, with a seeing disk between $1.5^{\prime \prime}$ and $3.5^{\prime \prime}$ (FWHM) for most objects, but up to $\simeq 6^{\prime \prime}$ for three of them. The log of the observations is given in Table 1, which is proposed in electronic form only. 
Table 1. Catalog elements and kinematical results

\begin{tabular}{|c|c|c|c|c|c|c|c|c|c|c|c|c|c|}
\hline Object & Type & 1950 & $\delta_{1950}$ & $B_{\mathrm{T}}$ & $-M_{B}$ & $r_{\mathrm{e}}$ & $\epsilon$ & $\mathrm{PA}$ & ref. & $v_{\text {hel }}$ & $\overline{\sigma_{0}}$ & $V_{\max }$ & $r_{\text {max }}$ \\
\hline (1) & (2) & (3) & $(4)$ & $(5)$ & (6) & $(7)$ & $(8)$ & (9) & (10) & (11) & $(12)$ & (13) & $\overline{(14)}$ \\
\hline IC 1211 & -5 & 51538.6 & -530740 & 3.49 & 20.72 & 18.3 & 0.13 & 57 & 1 & $563 \pm 29$ & $78 \pm 44$ & \pm & \\
\hline IC 4051 & -5 & 5830.1 & +281641 & 4.22 & 20.51 & 13.1 & 0.16 & 99 & 1 & $12 \pm 29$ & $233 \pm 37$ &.$\pm \ldots$ & \\
\hline NGC 0080 & -3 & 001835.1 & +220450 & 12.78 & 21.29 & 33.4 & 0.08 & $\ldots$ & 3 & $5689 \pm 22$ & $220 \pm 17$ & $15 \pm \ldots$ & \\
\hline NGC 0194 & -5 & 003644.0 & +024542 & 12.95 & 20.80 & 22.2 & 0.11 & 38 & 1 & $5183 \pm 20$ & $215 \pm 22$ & $39 \pm 18$ & 10 \\
\hline NGC 0410 & -4 & 010811.6 & +325309 & 12.18 & 21.59 & 37.0 & 0.29 & 34 & 1 & $5250 \pm 25$ & $277 \pm 21$ & $63 \pm 28$ & 15 \\
\hline NGC 0533 & -5 & 012257.0 & +013000 & 12.34 & 21.68 & 40.6 & 0.27 & 52 & 1 & $5466 \pm 33$ & $258 \pm 27$ & $\ldots \pm \ldots$ & \\
\hline NGC 2476 & -5 & 075322.7 & +400346 & .29 & 20.24 & 11.5 & 0.29 & 153 & 1 & \pm 18 & \pm 19 & $33 \pm 42$ & 14 \\
\hline NGC 2778 & -5 & $\begin{array}{lll}09 & 09 & 19.2\end{array}$ & +351359 & 13.43 & 18.90 & 14.1 & 0.19 & 46 & 1 & $2 \pm 22$ & $147 \pm 16$ & $130 \pm 33$ & 12 \\
\hline NGC 2872 & -5 & 092300.6 & +113856 & 12.54 & 20.74 & 24.0 & 0.20 & 148 & 1 & $3241 \pm 14$ & $288 \pm 14$ & $>62 \pm \ldots$ & 11 \\
\hline NGC 2874 & 4 & 092305.5 & +113831 & 12.27 & 21.23 & 25.4 & 0.67 & 43 & 3 & \pm 11 & $139 \pm 11$ & $\ldots \pm \ldots$ & \\
\hline NGC 3070 & -5 & 095527.0 & +103601 & 13.12 & 21.14 & 14.9 & 0.13 & 51 & 1 & \pm 17 & $239 \pm 16$ & $\ldots \pm \ldots$ & \\
\hline 31 & -5 & 101052.6 & +390047 & 12.37 & 22.31 & 46.8 & 0.11 & 158 & 1 & \pm 34 & \pm 21 & $129 \pm 15$ & 7 \\
\hline NGC 3226 & -5 & 102043.5 & +200906 & 12.05 & 19.62 & 44.1 & 0.20 & 6 & 1 & $1295 \pm 16$ & $183 \pm 20$ & $>43 \pm \ldots$ & 12 \\
\hline NGC 3245 & -2 & 102429.9 & +284547 & 14.44 & 19.98 & 22.1 & 0.32 & 177 & 3 & \pm 15 & $211 \pm 19$ & $147 \pm 27$ & 13 \\
\hline NGC 3599 & -2 & 111249.1 & +182308 & 12.67 & 18.81 & 29.9 & 0.00 & $\cdots$ & 3 & $835 \pm 22$ & $79 \pm 18$ & $\ldots \pm \ldots$ & \\
\hline NGC 3605 & -5 & 111408.7 & +181726 & 13.44 & 18.04 & 11.3 & 0.41 & 21 & 1 & $668 \pm 19$ & $94 \pm 28$ & $\ldots \pm \ldots$ & \\
\hline NGC 4111 & -1 & 120430.5 & +432043 & 11.58 & 19.06 & 10.2 & 0.75 & 150 & 2 & $779 \pm 12$ & $152 \pm 05$ & $160 \pm 08$ & 32 \\
\hline NGC 4239 & -5 & 121442.3 & +164834 & 13.69 & 17.43 & 14.7 & 0.48 & 115 & 1 & $940 \pm 16$ & $57 \pm 33$ & $\ldots \pm \ldots$ & 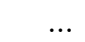 \\
\hline NGC 4318 & -5 & 122010.6 & +082832 & 14.15 & 16.86 & 7.4 & 0.33 & 67 & 1 & $1227 \pm 13$ & $77 \pm 17$ & $\ldots \pm \ldots$ & $\cdots$ \\
\hline NGC 4365 & -5 & 122155.0 & +073543 & 10.35 & 20.66 & 88.3 & 0.22 & 40 & 1 & $1252 \pm 17$ & $280 \pm 12$ & $41 \pm 14$ & \\
\hline NGC 4458 & -5 & 122625.9 & +133109 & 12.90 & 18.14 & 20.4 & 0.33 & 7 & 1 & $671 \pm 11$ & $116 \pm 15$ & $14 \pm 09$ & \\
\hline NGC 5129 & -5 & 132141.9 & +141411 & 12.83 & 21.97 & 30.6 & 0.30 & 5 & 1 & $6843 \pm 29$ & $252 \pm 31$ & $169 \pm 50$ & 9 \\
\hline NGC 6702 & -5 & 184530.9 & +453902 & 12.78 & 21.00 & 32.7 & 0.20 & 65 & 3 & $4727 \pm 10$ & $184 \pm 11$ & $<30 \pm \ldots$ & 18 \\
\hline NGC 7391 & -5 & 224802.1 & -014829 & 12.68 & 20.06 & 22.6 & 0.13 & 104 & 1 & $3038 \pm 25$ & $252 \pm 26$ & $<30 \pm \ldots$ & 15 \\
\hline NGC 7768 & -5 & 234826.2 & +265209 & 12.80 & 22.02 & 39.6 & 0.29 & 65 & 1 & $8050 \pm 30$ & $268 \pm 32$ & $105 \pm 29$ & 10 \\
\hline
\end{tabular}

Notes. Col. (2): morphological type (from the LEDA database - status: LEDA1996); Cols. (3), (4): coordinates, from Prugniel \& Simien (1997: hereafter PS97), except for NGC 2874 (from LEDA); Col. (5): $B_{\mathrm{T}}$, integrated blue magnitude, corrected for Galactic extinction and $k$ term (from PS97, except for NGC 2874 and NGC 3245: from LEDA); Col. (6): $M_{B}$, absolute $B$ magnitude (for a distance modulus from PS97, corresponding to $H_{0}=75 \mathrm{~km} \mathrm{~s}^{-1} \mathrm{Mpc}^{-1}$; except for NGC 2874 and NGC 3245: from LEDA); Col. (7): $r_{\mathrm{e}}$, effective radius, in arcsec (from PS97, except for NGC 2874 and NGC 3245: from Prugniel \& Héraudeau 1998); Col. (8): $\epsilon$, ellipticity; Col. (9): PA, position angle of major axis, in degrees (North through East, 0 to $180^{\circ}$ ); Col. (10): reference for $\epsilon$ and PA, $1=$ Djorgovski (1985), $2=$ Michard \& Marchal (1993), 3= LEDA; Col. (11): $v_{\text {hel }}$, heliocentric radial velocity, in $\mathrm{km} \mathrm{s}^{-1}$; Col. (12): $\sigma_{0}$, central velocity dispersion, in $\mathrm{km} \mathrm{s}^{-1}$; Col. (13): $V_{\max }$, maximum rotation velocity, in $\mathrm{km} \mathrm{s}^{-1}$ (the $>$ sign indicates that the rotation is still rising at the outermost point, or that the PA of the slit was inclined with respect to the major axis); Col. (14): $r_{\max }$, the radius at which $V_{\max }$ was measured, in arcsec.

\section{Data reduction}

As in Papers I to III, standard pre-processing was applied to the raw data, up to the rebinning in wavelength. The galaxy centers $(r=0)$ were determined by a Gaussian fitting to a limited range $\left(\simeq 12^{\prime \prime}\right)$ around the intensity peak. In the outer regions, cosmic-ray hits were removed with a median filter, and adjacent lines were combined with a variable weighting function (a Gaussian continuously wider faintward). A Fourier-Fitting technique determined the central velocity dispersion $\sigma_{0}$ and, when possible, the radial profile $\sigma(r)$ of the dispersion, together with the projected rotation curve $V(r)$ along the major axis. A two-pass mode (described in Paper I) allowed to remove cosmics on the inner lines, where the spatial resolution must be preserved. We adopted as the systemic velocity the value measured at $r=0$. Whenever possible, we have determined the maximum rotation velocity $V_{\max }$, as the mean of representative values on the opposite semi-axes.

\section{Presentation of the results}

Determinations of the heliocentric radial velocity $v_{\text {hel }}$, of $\sigma_{0}$, and of $V_{\max }$ (together with the corresponding radius $\left.r_{\max }\right)$ are listed in the last columns of Table 1 . The $V(r)$ and $\sigma(r)$ profiles are presented in Fig. 1, and also in Table 3 , which is proposed in electronic form only. As in Paper III, we have adopted the following convention for the position angle $\mathrm{PA}$ : for $0<\mathrm{PA}<180^{\circ}, r<0$ corresponds to the eastern side of the galaxy, for $180<\mathrm{PA}<360^{\circ}, r<0$ corresponds to the western side, and for $\mathrm{PA}=0^{\circ}, r<0$ is to the North. Tables 1 through 3 are available from the CDS.

Our results are summarized as follows: 

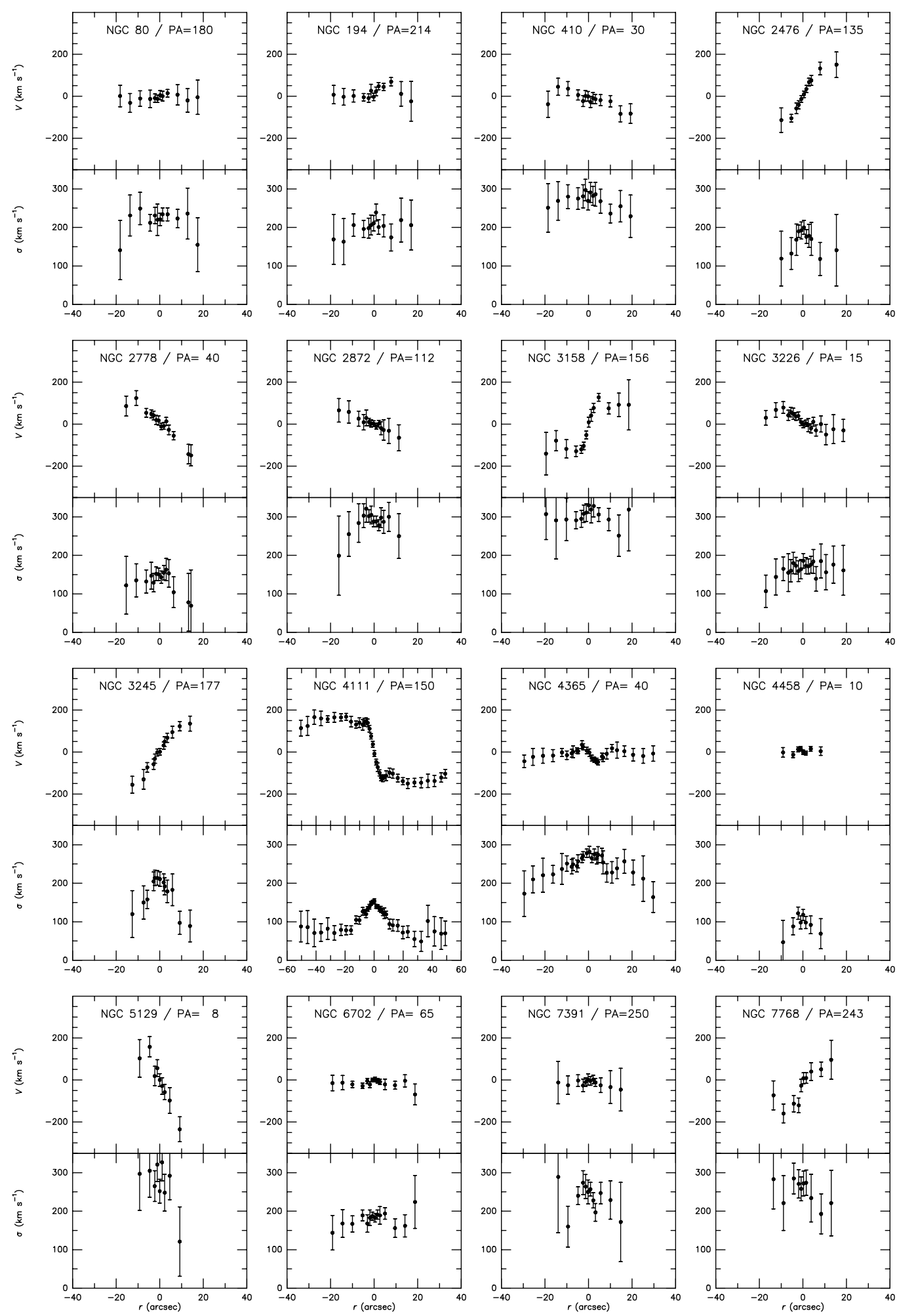

Fig. 1. Profiles of rotation velocities and velocity dispersions 
- We have measured the central velocity dispersion $\sigma_{0}$ for 25 galaxies: $20 \mathrm{Es}$, four S0s, and a spiral.

- For 15 galaxies in our sample, we have been able to determine the $\sigma(r)$ and $V(r)$ profiles along the major axis and, for another one, along an intermediate axis. Whenever possible (for 16 galaxies), we have determined the maximum rotation velocity $V_{\max }$ : this parameter was still unavailable for six of these objects.

The data presented here, together whith those of the preceding papers of the series, are available in the HYPERCAT on-line catalog browser (Prugniel et al. 1998), at http://www-obs .univ-lyon1.fr/hypercat.

Acknowledgements. We are endebted to the telescope operators at the Observatoire de Haute-Provence for their help in collecting the data. We thank the referee, G. Busarello, for his careful correction of the first version of the manuscript. We have made use of the Lyon-Meudon Extragalactic Database operated by the LEDA team.

\section{References}

Djorgovski S., 1985, PhD thesis. Univ. of California, Berkeley Michard R., Marchal J., 1993, A\&AS 98, 29

Prugniel Ph., Héraudeau Ph., 1998, A\&AS 128, 299

Prugniel Ph., Simien F., 1997, A\&A 321, 111 (PS97)

Prugniel Ph., Zasov A., Busarello G., Simien F., 1998, A\&AS 127, 117

Simien F., Prugniel Ph., 1997a, A\&AS 122, 521 (Paper I)

Simien F., Prugniel Ph., 1997b, A\&AS 126, 15 (Paper II)

Simien F., Prugniel Ph., 1997c, A\&AS 126, 519 (Paper III) 